(median $640 \mathrm{ng} / \mathrm{dl}$ or $22.2 \mathrm{nmol} / \mathrm{l})$. The levels of testosterone and dihydrotestosterone in prostate tissue, however, did not change significantly during the trial. No significant effect of TRT was observed on the histology and gene-expression profile of the prostate gland, or on voiding symptoms, urinary flow and incidence of prostate cancer.

The authors conclude that 6 months of TRT, which efficiently normalizes serum androgen levels, does not have any harmful effect on the prostate gland. They emphasize, however, that studies with large sample sizes and extended treatment duration are required to establish the safety of TRT in aging men.

Original article Marks LS et al. (2006) Effect of testosterone replacement therapy on prostate tissue in men with lateonset hypogonadism: a randomized controlled trial. JAMA 296: 2351-2361

\section{D2-receptor imaging has limited use in patients with nonfunctioning pituitary adenoma}

Surgery is the preferred treatment for clinically nonfunctioning pituitary macroadenoma, but these tumors often recur. Since some of these tumors express dopamine D2 receptors, dopamine-agonist therapy has been explored as an alternative to surgery and as a postoperative treatment, with favorable albeit heterogeneous results; de Herder and colleagues, therefore, investigated whether single-photon-emission CT (SPECT) imaging with ${ }^{123}$-epidepride, a radiolabeled dopamine D2 receptor antagonist, could predict patients' responses to dopamine-agonist therapy.

Of 54 patients with clinically nonfunctioning pituitary adenoma who underwent SPECT, 18 patients (age range 32-86 years; 12 men) received dopamine-agonist therapy with quinagolide (150-300 $\mu$ g daily) or cabergoline (1-2 mg weekly, titrated as required), for 34-187 months (mean 7.5 years). Five patients had previously undergone surgery and one required surgery after dopamine-agonist therapy was initiated. The other 36 patients required surgery or refused dopamine-agonist therapy. Patients underwent pituitary MRI at baseline, after 3-6 and 12 months of treatment, and annually thereafter.

Mean tumor shrinkage was $30 \%$ in cabergolide-treated and quinagolide-treated patients, which suggested that both agents reduced the need for postoperative radiotherapy. Pituitary uptake of ${ }^{123}$-epidepride did not predict patients' responses to dopamineagonist therapy, although when tumor shrinkage was defined as a decrease of $>20 \%$ on MRI, there was a trend for shrinkage to correlate with uptake of ${ }^{123}$-epidepride, especially for tumors with moderate uptake.

The authors conclude that ${ }^{123}$-epidepride SPECT is most useful to differentiate scar tissue from recurrent or residual tumor.

Original article de Herder WW et al. (2006) Dopamine agonist therapy of clinically non-functioning pituitary macroadenomas. Is there a role for ${ }^{123}$ I-epidepride dopamine D2 receptor imaging? Eur J Endocrinol 155: 717-723

\section{Low plasma adiponectin levels are indicative of the metabolic syndrome}

Low plasma adiponectin levels have been suggested as a biomarker for the metabolic syndrome. The International Diabetes Foundation (IDF) has introduced new criteria to diagnose the metabolic syndrome; Santaniemi and colleagues, therefore, investigated whether the relationship between plasma adiponectin level and the metabolic syndrome was influenced by use of these new diagnostic criteria.

Santaniemi and colleagues' cross-sectional study used their own enzyme-linked immunosorbent assay to measure plasma adiponectin levels in 1,041 Finnish participants from the Oulu Project Elucidating the Risk of Atherosclerosis (a population-based epidemiologic study of cardiovascular risk factors).

The metabolic syndrome was diagnosed in 388 and 308 participants by the IDF and the old criteria, respectively. Individuals with the metabolic syndrome were markedly hypoadiponectinemic, irrespective of their sex or the diagnostic criteria used; low plasma adiponectin levels were associated with all features of the metabolic syndrome except free plasma glucose levels. Plasma adiponectin levels correlated inversely with the number of metabolic abnormalities, in both sexes. The adjusted probability of having the metabolic syndrome was highest in individuals with adiponectin levels in the lowest quartile: compared to those with adiponectin levels in the 